\title{
Age and metallicity of Galactic clusters from full spectrum fitting
}

\author{
Mina Koleva ${ }^{1,2} \dagger$, Philippe Prugniel ${ }^{1,3}$, Pierre Ocvirk ${ }^{4}$ and Damien \\ Le Borgne ${ }^{4}$ \\ ${ }^{1}$ Université Lyon 1, Observatoire de Lyon, St. Genis Laval, 69230, France; CNRS UMR 5574 \\ ${ }^{2}$ Department of Astronomy, St. Kl. Ohridski University of Sofia, BG-1164 Sofia, Bulgaria \\ ${ }^{3}$ Observatoire de Paris, GEPI, F-75014, France \\ ${ }^{4}$ CEA Saclay/Service d'Astrophysique, Gif-sur-Yvette Cedex, F-91191, France
}

\begin{abstract}
We are using full spectrum fitting to determine the ages and metallicities of Galactic clusters (M67 and globular clusters). We find that the method is very accurate to measure the metallicity. Blue horizontal branches are well identified as a 'young' sub-component, and the age of the 'old' component is in fair agreement with CMD determinations.
\end{abstract}

Keywords. globular clusters: general; galaxies: star clusters; techniques: spectroscopic

Goal. The spectroscopic determination of age and metallicity of star clusters is a key to understand the characteristics and origin of extragalactic cluster systems. It is also a challenge for analysis methods. The goal of this work is to analyze spectra of Galactic clusters for which CMD measurements are independently available.

Observational material. We are using the spectrum of the open cluster M67 synthesized by Schiavon et al (2004) and the library of spectra of globular clusters assembled by Schiavon et al. (2005). The spectra have a resolution of about 3 Aand the analysis is performed in the optical range between $\mathrm{H} \& \mathrm{~K}$ and $\mathrm{H}_{\alpha}$.

Method. Since our goal is to study extragalactic clusters and dwarf galaxies, we are concerned by the optimal usage of the information. For this reason we use full spectrum fitting rather than spectroscopic indices. Another reason is that modern observations provide relatively high resolution spectra of clusters $(R=1000-5000)$ that would not be adequately exploited using indices. Still, as well as indices, our method is designed to be essentially insensitive to the shape of the continuum, so that the characteristics of the population are constrained by the relative depths and shapes of the spectral lines. The method consists in comparing observed spectra to population models generated with Pegase.HR (Le Borgne et al. 2004) using the library ELODIE.3.1 (in preparation, see Prugniel \& Soubiran 2001, 2004 for the previous versions). We are using two programs implementing this method: STECKMAP (Ocvirk et al. 2006), can constrain the history of the stellar population, and NBursts (Chilingarian, this conference) determines the SSP-equivalent parameters. Both programs measure simultaneously the broadening of the spectrum (kinematical and observational) and the characteristics of the population. The precision of the method has been discussed in Koleva et al. (2006) and consistency tests are presented in Koleva et al. (this conference).

Results. The quality of the fits is in general excellent, and does not appear to be limited by the model, except for high metallicity clusters where $\alpha$-element abundances exceed the

$\dagger$ mina.koleva@obs.univ-lyon1.fr 

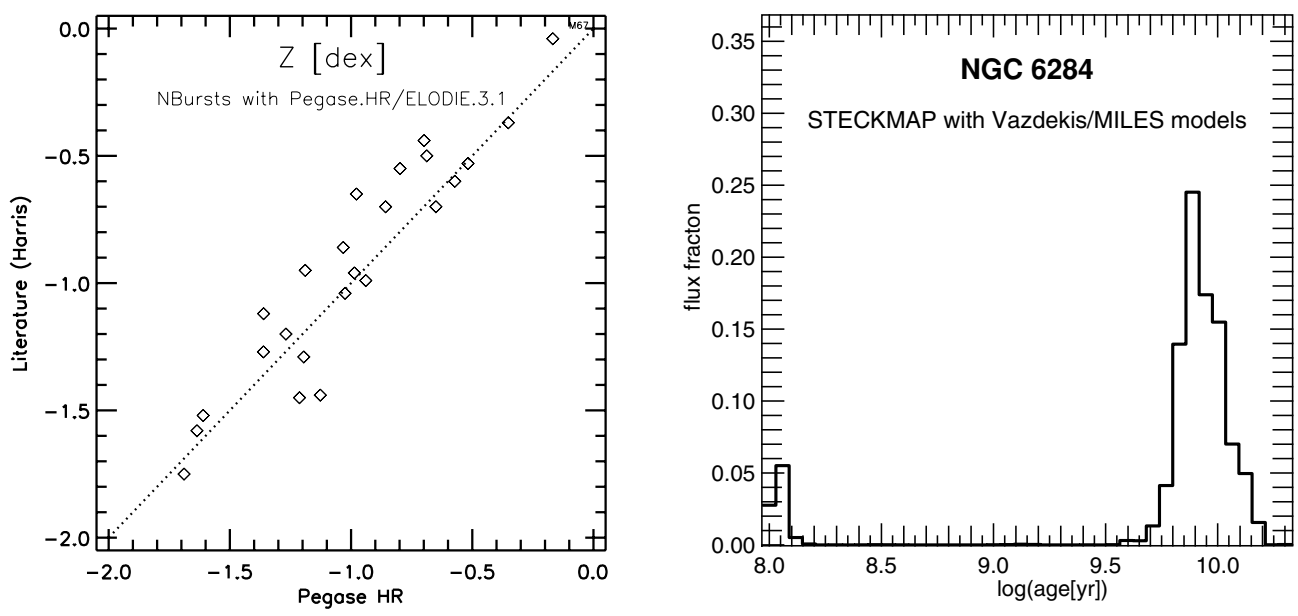

Figure 1. (a) Comparison between metallicity measured by spectrum fitting and literature.

(b) Detection of 'young' component in BHB clusters: case of NGC 6284.

models (see Prugniel et al., this conference). The SSP-equivalent metallicities (Fig. 1a) are in good agreement with CMD determination and have a precision is 0.15 dex.

The SSP-equivalent ages are strongly affected by the HB morphology: Clusters with BHB are found younger with NBursts. This excess of blue stars is detected as a 'young subcomponent' in the stellar age distributions reconstructed with STECKMAP (Fig. 1b). The age of the 'old' component agrees better with CMD fits than the SSP-equivalent age.

The ages of the old stellar systems are very sensitive to the temperature scale of the cold giants. A difference of $200 \mathrm{~K}$ changes the age estimate by 20 or $30 \%$.

Conclusion. Spectrum fitting of globular clusters robustly pinpoint BHBs (or blue stragglers), and it seems possible to fit a model with a BHB component.

Full spectrum fitting, with our method, is a reliable approach to find metallicities and ages of stellar systems over a wide range of parameters. Using the high spectral resolution models built with ELODIE.3.1 or with CFLIB (Valdes et al. 2004) will allow us to understand better the origin of extragalactic globular cluster systems.

Acknowledgements We thanks R. Schiavon for giving the M67 spectrum. MC acknowledges the financial support from IAU. DLB acknowledges the French CNES.

\section{References}

Harris, W.E. 1996, AJ, 112, 1487, (http://www.physics.mcmaster.ca/ ^harris/mwgc.dat)

Koleva, M., Bavouzet, N., Chilingarian, I., \& Prugniel, Ph, 2006, astro-ph/0602362

Le Borgne, D. et al., 2004, A\&A 425, 881

Ocvirk, P., Pichon, C., Lançon, A., \& Thiébaut, E., 2006, MNRAS 365, 74

Prugniel, Ph. \& Soubiran, C.,2001, A\& A, 369, 1048

Prugniel, Ph. \& Soubiran, C., 2004, astro-ph/0409214

Schiavon, R., Caldwell, N., \& Rose, J., 2004, AJ 127, 1513

Schiavon, R., Rose, J., Courteau, S., \& McArthur, L., 2005, ApJS 160, 163

Sánchez-Blázquez, P. et al., 2006, MNRAS 371, 701

Valdes, F. Gupta, R., Rose, J., et al. 2004, ApJS 152, 251 